\title{
Medical Image of the Week: Fat Embolism
}

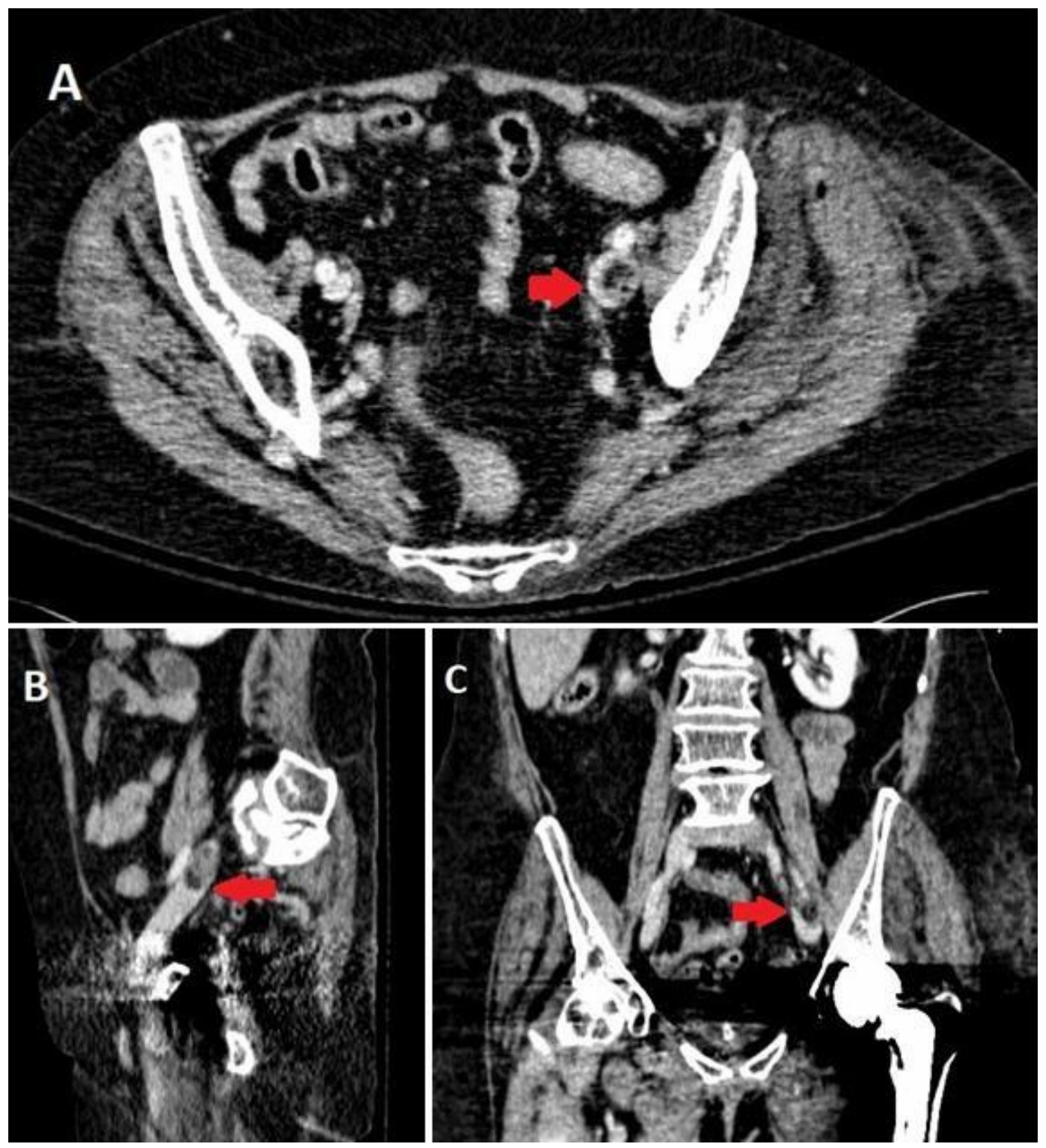

Figure 1. A CT with IV contrast at the pelvic level showing a filling defect (red arrow) in left external iliac vein extending from left common iliac vein in axial (A), sagittal (B), and coronal

(C) sections. Its attenuation coefficient (Hounsfield Unit) suggests fat embolus mixed with thrombus.

A 74-year-old woman presented to the hospital with a left femoral neck fracture after a fall. The next day she underwent an uneventful left hip hemiarthroplasty. About 3 hours postoperatively, she became lethargic, tachycardic, tachypneic, febrile, and hypotensive. An arterial blood gas analysis revealed mild hypoxemia with $\mathrm{PaO} 2$ / FiO2 ratio of 270 . Hemoglobin decreased from 
$9.4 \mathrm{~g} / \mathrm{dL}$ to $7.7 \mathrm{~g} / \mathrm{dL}$ postoperatively. A chest $\mathrm{x}$-ray showed only bibasilar opacities. Sepsis, acute postoperative blood loss anemia, and pulmonary embolism (PE) were entertained as a differential diagnosis. The patient was resuscitated with intravenous fluids and packed red blood cells, and was started on broad-spectrum antibiotics. Her hemodynamic status stabilized shortly thereafter. A CT chest with PE protocol and abdomen/pelvis down to thigh level was performed; no PE was identified. It did not show any intraabdominal pathology or signs of hemorrhage. However, it incidentally revealed fat embolus mixed with thrombus in the left common and external iliac veins (Figure 1). All microbiologic studies were negative. Therefore, she was thought to have fat embolism syndrome (FES) with transient systemic inflammatory syndrome (SIRS). An IVC filter was placed and anticoagulation was also started.

FES is a collection of symptoms and signs including respiratory insufficiency, petechial rash, and neurologic impairment associated with fat in the circulation (1). Fat emboli cause an intense inflammation (2), and FES shares many features characteristic of systemic inflammatory response syndrome (SIRS) (1). It is a diagnostic challenge as many of the manifestations are common to other critical illnesses (1). It is most commonly associated with long bone fractures and orthopedic procedures. Literature on radiographic finding of fat emboli in the venous system is rare (3); although neither necessary nor sufficient, its radiographic demonstration in the venous system can substantially aid in diagnosis of FES, as demonstrated in our case.

Hyeong J. Kim MD'1, Michael Jesinger MD², and Medhi Khosravi MD²

1 Division of Pulmonary, Critical Care, and Sleep Medicine, East Carolina University, Greenville, NC, USA

2 Internal Medicine, University of Kentucky, Lexington, KY, USA

${ }^{3}$ Division of Pulmonary, Critical Care, and Sleep Medicine, Division of Pulmonary, Critical Care, and Sleep Medicine, Lexington, KY, USA

\section{References}

1. Mellor A, Soni N. Fat embolism. Anaesthesia. 2001 Feb;56(2):145-54. [CrossRef] [PubMed]

2. Kwiatt ME, Seamon MJ. Fat embolism syndrome. Int J Crit IIIn Inj Sci. 2013 Jan;3(1):64-8. [CrossRef] [PubMed]

3. Harris AC, Torreggiani WC, Lyburn ID, Zwirewich CV, Ho SG, Munk PL. CT and sonography of traumatic fat embolism in the common femoral vein. AJR Am J Roentgenol. 2000 Dec;175(6):1741-2. [CrossRef] [PubMed] 